\title{
RÉCENTES ACQUISITIONS ET PERSPECTIVES DE L'ENTOMOLOGIE MÉDiCALE ET DE LA LUTTE ANTIVECTORIELle ${ }^{1}$
}

\author{
J. MOUCHET*, C. BELLEC**
}

\begin{abstract}
RÉSUMÉ
Les technologies avancées de biologie moléculaire, génétique, informatique, sont à l'origine de progrès importants en taxonomie, physiologie, bioécologie et épidémiologie. De nouveaux insecticides, des IGR et des bactéries entomopathogènes ont été développés. Les succès de la lutte antivectorielle se partagent entre moyens simples (pièges à tsé-tsé et moustiquaires imprégnées) et les programmes lourds (lutte contre l'onchocercose en Afrique de l'Ouest, démoustication dans l'hémisphère nord). La lutte contre la dra-

cunculose se situe entre les deux. La lutte antipaludique a des difficultés à retrouver son second souffle. Les luttes biologique et génétique piétinent.

Des structures adéquates, du personnel bien formé et motivé, un financement convenable, une volonté politique restent les facteurs incontournables de l'emploi des méthodes actuellement disponibles.

Summary: Medical entomology and vector control: new findings and prospects.

High technologies of molecular biology and genetics, supported by computering brought considerable advances in taxonomy, physiology, host/parasite relationships, ecology and epidemiology. New insecticides, IGR and bacteria are now available. Both simple methods (e. g. tsetse trapping, impregnated bednets) and large programs using heavy material (e. g. O. C. P. in West Africa, mosquito control in Northern hemisphere) have been successfull.

Guineaworm control lies in an intermediate position. Malaria control has difficulties and needs new tools and a better use of those already existing. A few progresses are noticed in biological and genetical control.

Appropriate structures, well trained and motivated personnel, adequate funding and political willingness are the keys for the implementation of any control programme.
\end{abstract}

Pour réaliser ce travail nous avons consulté les revues d'analyses des 4 dernières années et adressé un questionnaire à 150 de nos collègues. Nous remercions tous ceux qui ont répondu.

\section{PROGRÈS RÉCENTS DANS LE DOMAINE DE L'ENTOMOLOGIE MÉDICALE}

\section{1 - TAXonomie, PHysiologie, BIOÉCOLOGIE}

Le fait marquant a été l'utilisation grandissante des technologies avancées (11). La morphologie, base de l'identification des vecteurs commence à bénéficier de l'appui de

${ }^{1}$ Un document plus étoffé sera délivré aux participants qui en feront la demande.

* Inspecteur général de recherches honoraire ORSTOM, ORSTOM, Département Santé, 213, rue Lafayette, F 75480 Paris Cedex 10.

** Directeur de recherches ORSTOM, Centre ORSTOM de Montpellier, laboratoire d'Épidémiologie des Grandes Endémies Tropicales, B. P. 5045, 34032 Montpellier Cedex 1, France. l'informatique qui intègre des données écobiologiques. La cytogénétique, la génétique moléculaire (isoenzymes, sondes d'ADN) ont permis l'analyse des complexes d'espèces. L'analyse des hydrocarbones cuticulaires a été appliquée à la taxonomie des anophèles, simulies et glossines, celle des phéromones aux phlébotomes.

Des anticorps monoclonaux et des sondes d'ADN sont disponibles pour la recherche et l'identification chez les vecteurs, des plasmodies, des leishmanies, des trypanosomes et des filaires. Les techniques d'amplification des acides nucléiques (P. C. R.) permettent la détection, chez les vecteurs, de parasites présents en très faible quantité.

Les travaux sur la reproduction et le développement, le déterminisme de l'autogénèse, la détermination de l'âge physiologique par les ptéridines, les phéromones et les comportements sexuels sont à retenir. Ceux sur les attractifs olfactifs et sur les comportements de recherche de l'hôte sont menés pour améliorer les méthodes de lutte (2).

Les études enzymatiques et génétiques sur la résistance aux insecticides visent à détecter précocement le phénomène et à déterminer ses mécanismes.

Dans le domaine des rapports vecteurs/parasites (6) on 
a noté le rôle des lectines et des RLO (rickettsia like organisms) dans le cycle de développement des trypanosomes chez les glossines. La reproduction sexuée des leishmanies chez les phlébotomes a été établie. Par manipulations génétiques, on a introduit un ADN étranger dans le génome d'Anopheles gambiae et sélectionné une lignée totalement réfractaire à Plasmodium falciparum.

Les modifications écologiques, anthropiques ou naturelles de l'environnement se répercutent sur la distribution et la dynamique des populations de vecteurs (8). La déforestation entraîne la pullulation des espèces héliophiles et amène l'homme au contact de foyers sylvatiques de zoonoses. Les grands travaux, barrages et périmètres irrigués, entraînent la pullulation des culicidés. L'urbanisation pollue les eaux de surface. L'emploi des pesticides agricoles favorise les vecteurs résistants. Le réchauffement de la terre pourrait modifier la répartition des espèces et leur pouvoir vecteur.

L'étude des vecteurs de loase au Congo a montré les différences écologiques entre Chrysops silacea et $C$. dimidiata qui sont sans contact avec les singes ainsi exclus du cycle de la maladie. En Amérique du Sud, les réduviidés péridomestiques ont tendance à occuper les maisons après l'élimination des espèces domestiques par les insecticides (1). Les études des foyers de leishmaniose, au Maroc et en Bolivie, conduisent au concept d'une biogéographie parasitaire.

Les moyens de communication ont pris un essor tel qu'aucun pays ne peut se dire à l'abri de l'importation d'un vecteur ou d'un parasite (5). Aedes albopictus vient d'être introduit aux États-Unis, Cochliomyia hominivorax, en Lybie.

Le paludisme des aéroports en Europe est consécutif à l'importation d'anophèles africains qui n'ont pas fait souche car introduction ne signifie pas implantation durable. Ce point doit être pris en compte dans l'évaluation des risques liés à la construction d'une route transaharienne.

\section{2 - ÉPIDÉmiologie des maladies À vecteurs}

Les arthropodes peuvent être exclus de l'épidémiologie du SIDA (7). Les risques de transmission par repas interrompu de l'insecte, seuls envisageables, sont inférieurs à 1 sur 10 millions. L'épidémiologie confirme ces vues étant donné l'absence de cas entre 2 et 12 ans.

La transmission épizootique de la Rift Valley Fever par Aedes lineatopennis semble liée à l'inondation de dépressions asséchées depuis une ou plusieurs années. La télédétection est utilisée pour dépister les zones à risque par l'indicateur de végétation. Les épidémies selvatiques de Fièvre Jaune, observées au Ghana, Burkina Faso, Mali, Guinée, Nigéria (pro parte), confirment les schémas de maintien et de dissémination du virus en période interépidémique.

La maladie de Lyme (12) à Borrelia burgsdorferi découverte aux États-Unis est fréquente dans les régions tempé- rées de l'Eurasie. Sa répartition suit celle de ses vecteurs les Ixodes du groupe ricinus. Leurs larves vivent sur les rongeurs, surtout mulots et campagnols réservoirs de la Borrelia, les nymphes et les adultes se fixent sur les ruminants sans symptômes et sur l'homme qui fait une maladie sévère.

Sur la côte de Nouvelle Angleterre aux États-Unis on observe des cas humains de Babesia microti, parasite des rongeurs myomorphes, transmis par Ixodes dammini.

En 1990, le paludisme est un risque pour près de la moitié de la population du globe. On estime en 1988 à plus de 100 millions le nombre des cas en Afrique et à 5 millions pour le reste du monde. L'objectif de la lutte antipaludique étant la réduction de la mortalité et de la morbidité spécifiques, les études épidémiologiques (3.8) tentent de relier la transmission aux manifestations de la maladie : Ces études ont montré : $a$ ) l'hétérogénéité épidémiologique du paludisme même à l'intérieur des zones où il est très stable comme en Afrique tropicale, $b$ ) l'absence de corrélation entre le taux d'inoculation et les manifestations pathologiques de la maladie dans les zones de haute endémicité, du fait du développement concomittant de l'immunité, $c$ ) le risque épidémique considérable dans les zones d'instabilité, $d$ ) les facteurs locaux de variation apportés par les grands cours d'eau ou les lagunes saumâtres ainsi que par les modifications anthropiques du réseau hydrographique et par l'urbanisation.

L'irrigation, facteur de prolifération des moustiques, peut soit générer le paludisme (Burundi) soit être neutre, voire même diminuer la transmission (Burkina Faso) suivant la situation épidémiologique. Le paludisme urbain, réalité en Asie du Sud-Est, est en général, en Afrique tropicale, une transposition atténuée du paludisme rural.

Le typage des leishmanies chez les phlébotomes a montré l'étroite association de chaque leishmanie à un ou des sous genres de phlébotomes suite à une co-évolution parasite/vecteur (4). Après typage, de nouveaux vecteurs de L. (Vianna) braziliensis ont été identifiés en Bolivie et de L. mexicana en République Dominicaine; d'autres ont été remis en question en Eurasie.

Les comportements humains et l'occupation de l'espace, modulateurs de contact homme/glossine en Côte d'Ivoire, sont la clef de voûte de l'épidémiologie de la maladie du sommeil.

L'absence de caractères indiscutables pour séparer les populations d'onchocerques de savane (cécitantes) et de forêt, dermotrope à leur stade adulte ou à leur stade de larve infectante (L3), est un handicap pour les études épidémiologiques conduites en Afrique. La transmission dans les foyers de l'Équateur et de la frontière Venezuela-Brésil (Sierra Parima) a nécessité une clarification taxonomique des vecteurs.

Au Congo, le potentiel annuel de transmission de la loase (150-200 L3) établi d'après des captures cumulées des 
espèces vectrices a été relié à la prévalence de la maladie chez les populations bantous et pygmées.

L'écologie de la dizaine d'espèces de Cyclops impliqués dans les cycles de la dracunculose, en Afrique de l'Ouest et Centrale, commence seulement à être étudiée dans la perspective de la lutte. Les taux d'infection des Cyclops peuvent atteindre $20 \%$, le nombre de L3 par specimen étant de 3 à 5 .

L'entomologiste médical ne peut ignorer les arthropodes non vecteurs, qui constituent des nuisances par leurs piqûres ou leur simple présence. En effet : $a$ ) dans les pays industrialisés ils font l'objet de campagnes de lutte dotées de gros moyens techniques et assorties de recherches, sources de progrès techniques, $b$ ) dans les pays pauvres comme dans les pays riches ils sont la cible de la désinsectisation domestique, protection non négligeable de la population, c) ils motivent les mesures de protection individuelle, $d$ ) leur disparition est pour la population le critère d'efficacité de la lutte antivectorielle.

\section{LUTTE ANTIVECTORIELLE}

\section{1 - PRINCIPES ET OBJeCtifs}

Étymologiquement la lutte antivectorielle ne concerne que les vecteurs de maladie mais en fait elle recouvre également la lutte contre les insectes nuisants.

La protection contre les vecteurs et leur destruction constituent des mesures de prévention, complémentaires de l'utilisation de médicaments et de vaccins, et s'intègrent dans une stratégie générale d'attaque de la maladie. Leur place, en regard des autres moyens de lutte, dépend de leur efficacité respective ainsi que du contexte épidémiologique, de leur coût et des ressources de chaque pays.

Les stratégies se modifient avec l'apparition de nouveaux médicaments, insecticides, techniques, ainsi qu'avec le développement des résistances des vecteurs aux insecticides et des parasites aux médicaments. Les changements des politiques de santé et des structures sanitaires, l'évolution économique, le comportement des populations, les considérations éthiques et environnementalistes infléchissent également les choix.

\section{2 - Évaluation}

L'évaluation de la lutte antivectorielle au plan entomologique, c'est-à-dire en terme de réduction des populations de vecteurs et de leurs contacts avec l'homme, est une étape nécessaire, suffisante lorsqu'il s'agit seulement de contrôler des nuisances, mais insuffisante dans les actions de santé publique. La réduction de la transmission n'est aussi qu'une étape intermédiaire avant l'évaluation décisive qui est l'impact des traitements sur l'incidence de la maladie. L'évaluation est donc pluridisciplinaire.

\section{3 - Les nouveaux outils De LUTTE}

Des nouveaux produits : des pyréthrinoides photostables et des carbamates ont été commercialisés. Malgré les progrès des formulations de Bacillus thuringiensis $\mathrm{H} 14$ la rémanence reste toujours faible. $B$. sphaericus constitue un alternatif contre les populations multirésistantes de Culex quinquefasciatus. Malgré des performances prometteuses, de nombreux inhibiteurs de croissance n'ont pas été commercialisés.

Des nouvelles méthodes. Le recouvrement de la surface des puisards et latrines inondées, par des billes de polystyrène en couche continue empêche la ponte et le développement de Culex quinquefasciatus.

Le piège à glossines à attraction optique de ChallierLaveissière, amélioré par l'imprégnation avec un pyréthrinoide photostable, a donné naissance à des variantes comme le piège pyramidal de Lancien et Gouteux, utilisable éventuellement sans insecticide, et les écrans imprégnés (2).

Les moustiquaires (10) sont utilisées depuis longtemps mais leur efficacité est compromise par leur mauvaise utilisation et leur entretien défectueux. Leur imprégnation par des pyréthrinoides stables, deltaméthrine et perméthrine, qui persistent pendant une année, a éliminé ces inconvénients et en a fait non seulement des moyens de protection individuelle mais encore de réels outils de lutte lorsque leur emploi est généralisé au sein d'une communauté. L'imprégnation est bon marché et simple, exécutable par la population avec une supervision des techniciens de santé. De ce fait elle peut être continuée indéfiniment et le problème éthique de l'arrêt des traitements est minimisé. Les facteurs limitant leur emploi sont : $a$ ) l'achat des moustiquaires, b) leur acceptation par les habitants là où ils ne sont pas motivés par la nuisance; $c$ ) le développement possible de résistance aux pyréthrinoides.

Dans la lutte biologique hormis les bactéries entomopathogènes déjà citées, il n'y a guère que les poissons larvivores qui soient opérationnels. Encore faut-il préciser que bien qu'employés depuis 60 ans, leur impact réel n'a pas été évalué en terme de santé publique sauf dans des situations spécialement favorables. Les agents fongiques, les Mermis, les Cyclops ne sont toujours pas opérationnels.

Les espoirs mis dans les lâchers des mâles stériles ne se sont guère concrétisés au plan opérationnel. Le remplacement des vecteurs par des souches non vectrices de la même espèce, se heurte aux difficultés d'une introduction compétitive sur le terrain.

On a pu introduire en laboratoire un gêne cloné de Bacillus thuringiensis chez des Cyanobactéries pour leur faire produire directement le bacille dans le milieu naturel. Le passage au terrain est loin d'être réalisé et pose les problèmes d'éthique propres à toute manipulation génétique du milieu. 


\section{4 - Aménagement du Milieu}

L'élimination des eaux stagnantes, une des plus anciennes mesures de prophylaxie antipalustre, a été reprise dans des projets d'aménagement dotés d'engins lourds et plus récemment dans la promotion de l'hygiène villageoise, par la participation communautaire. D'un strict point de vue de santé publique on peut s'interroger sur leur rapport coût/efficacité. Trois expériences ont été tentées en Inde pour évaluer le potentiel de ces mesures, contre les anophèles et le paludisme dans le Gujerat, contre les moustiques urbains à Pondichery, contre les vecteurs de Brugia malayi dans le Kerala.

Le forage de puits associé au filtrage des eaux de boissons et à l'éducation sanitaire a entrâné une réduction voire une élimination de la dracunculose en Inde. En Afrique de l'Ouest l'éradication de la maladie est envisagée.

L'aménagement de l'environnement doit être fait avec prudence pour éviter de jouer les apprentis sorciers. Par exemple, la destruction des galeries forestières, pratiquée avec succès pour l'élimination des glossines riveraines en Afrique de l'Ouest est maintenant considérée comme inacceptable, la préservation de ces formations étant une priorité écologique. L'autre face du problème est la modification profonde de l'environnement par l'anthropisation (cf. 1.2.).

\section{5 - LES OPÉRATIONS DE LUTTE ANTIVECTORIELle}

D'après nos collègues les opérations jugées réussies ou prometteuses sont :

Le Programme de lutte contre l'onchocercose (9) en Afrique de l'Ouest (OCP) qui recueille l'unanimité des suffrages. Cette action supranationale exécutée dans une structure verticale avec un financement international, a obtenu des résultats remarquables puisque la parasitose a disparu dans $80 \%$ de la surface protégée et que de nombreuses vallées ont été repeuplées. Les raisons du succès sont, $a$ ) une excellente préparation scientifique et technique, une organisation efficace qui a permis, lorsque nécessaire, des modifications rapides de tactique, $b$ ) un système permanent d'évaluation pour détecter toute faille des opérations, $c$ ) une structure de recherches propre et un appel constant à l'expertise extérieure pour trouver des solutions à tous les problèmes. OCP a ainsi pu faire face avec succès aux réinvasions de simulies par traitement des rivières productrices, et aux résistances des vecteurs par l'utilisation en rotation d'une gamme d'insecticides préalablement sélectionnés.

Les campagnes de démoustication en Europe et en Amérique du Nord sont basées sur une parfaite connaissance du milieu (carte écologique) et une utilisation rationnelle des insecticides et des bactéries entomopathogènes. Elles s'appuient sur des structures techniquement et administrativement solides et leurs financements sont assurés par les collectivités protégées.
Les campagnes de lutte par piégeage contre les glossines (2) du groupe G. palpalis, menées avec des moyens modestes et la participation des populations. Elles ont permis d'arrêter la transmission dans des foyers de maladies du sommeil en Côte d'Ivoire et en Ouganda (cf. supra). Il faut signaler la part déterminante prise par quelques chercheurs motivés pour mobiliser et les communautés et les autorités sanitaires.

Mais ces succès ne doivent pas masquer les problèmes. Le paludisme, par exemple est en expansion, suite au relâchement ou à l'abandon des mesures de prévention et au développement des résistances aux médicaments. La lutte antivectorielle redevient d'actualité. On dispose pour la mener : a) des traitements intradomiciliaires, efficaces à condition d'assurer une bonne couverture de la population avec des insecticides appropriés. Ils sont onéreux, leur application demande des structures bien organisées et ils sont peu performants dans les zones de savanes d'Afrique. Leur application prolongée a découragé les exécutants, les populations et les bailleurs de fonds. Leur intégration dans les SSP n'a pas eu de succès. $b$ ) déjà mentionnées, les moustiquaires imprégnées ont montré leur potentiel en Chine. $c$ ) la lutte antilarvaire par des agents chimiques ou biologiques ainsi que par des aménagements de l'environnement est d'emploi limité.

Il ne fait pas de doute que de nouveaux outils seraient les bienvenus mais les recherches en cours ne laissent guère espérer de miracle dans un proche avenir. Il faut donc faire le meilleur usage de ceux dont on dispose. Ils sont suffisants pour passer à l'action dans nombre de situations.

\section{6 - Les CONDitions DE Mise EN CEUVRE DE LA LUTTE ANTI- VECTORIELLE}

Les études de base sont nécessaires pour : $a$ ) connaître la biologie des vecteurs concernés et leur sensibilité aux insecticides, $b$ ) évaluer au plan entomologique et épidémiologique les méthodes de lutte envisagées et tester leur acceptabilité par les populations, $c$ ) déterminer le coût et la durée probable des actions prévues, $d$ ) faire un inventaire des structures et des personnels, $e$ ) recenser les ressources financières disponibles localement et les besoins d'aides extérieures, $g$ ) en fonction de ces éléments déterminer la stratégie à appliquer.

L'éxécution de la lutte elle-même repose sur : $a$ ) une volonté politique, $b$ ) l'existence ou la création de structures adaptées à l'exécution des mesures envisagées, $c$ ) un personnel bien formé à tous les niveaux, $d$ ) des dotations financières suffisantes et prévues pour toute la durée des programmes.

Au plan éthique : il faut envisager les risques épidémiques en cas de cessation des opérations et les dangers des manipulations de l'environnement. 


\section{PERSPECTIVES}

L'entomologie médicale est une discipline carrefour qui doit à la fois utiliser les technologies les plus avancées et prendre en compte les réalités du terrain. Son aspect pluridisciplinaire, évident avec l'épidémiologie, doit se développer vers les sciences fondamentales (génétique et biologie moléculaire) en amont, vers l'écologie, la géographie, l'ethnosociologie voire l'agronomie et l'économie en aval. Le remodelage de la planète par les activités humaines introduira de nouvelles données épidémiologiques à prendre en compte pour la gestion de l'environnement, dans une optique intersectorielle.

Plus que jamais il faut établir un double flux entre laboratoire et terrain sur un plan de partenariat loyal. Les chercheurs de terrain sont en voie de disparition, en particulier dans les pays en développement, où les positions universitaires sont plus valorisantes que la prospection dans des marigots. Cette hiérarchisation élitiste des activités se ressent au niveau des organismes dispensateurs de crédits qui accordent peu de considération aux projets d'où les technologies avancées sont absentes.

Le potentiel de la lutte antivectorielle est sous-employé et nombre de méthodes actuellement disponibles ne sont pas utilisées. Dans l'immédiat les pays d'endémie, généralement pauvres, souhaitent des techniques de lutte simples et peu onéreuses qui sont quelquefois le fruit d'années de recherches. Mais, grâce à l'aide internationale, même ces pays peuvent envisager des programmes lourds utilisant des technologies avancées. OCP en est le meilleur exemple.

Le désir d'obtenir des résultats rapides ne doit pas faire rejeter les recherches à long terme, même très sophistiquées, pouvant déboucher sur de nouvelles méthodes de lutte. Il faut laisser la porte ouverte à la créativité.

Il existe un besoin d'entomologistes médicaux. Une formation large, que peu d'instituts sont actuellement capa- bles de dispenser, devrait précéder une spécialisation plus étroite. Il faut aussi se préoccuper du suivi des personnels formés pour éviter leur perte d'expertise.

Des structures adéquates, du personnel bien formé et motivé, un financement convenable, une volonté politique, restent les facteurs incontournables pour le développement de la lutte antivectorielle.

\section{RÉFÉRENCES}

1. Brenner R. R. et al. : Chagas' disease vectors. Volume I. Taxonomic, ecological, and epidemiological aspects. CRC Press Inc., Boca Raton, Florida, 1987, 155 p.

2. Cuisance D. : Le piégeage de Tsé-tsé. Études et synthèses de l'I. E. M. V. T., 1989, 32, 172 p.

3. Haworth J. : Malaria in man: its epidemiology, clinical aspects and control. A review of recent abstracts. Trop. Dis. Bull., 1989, 86, $66 \mathrm{p}$.

4. Killick-Kendrick R. : Phlebotomine vectors of the leishmaniasis: a review. Med. Vet. Ent., 1990, 4, 1-24.

5. Laird M. (Editor) : Commerce and the spread of pests and disease vectors. Praeger Publishers, New York, 1984, 354 p.

6. Maudlin I., Welburn S. C. : Tsetse immunity and the transmission of trypanosomiasis. Parasitol. Today, 1988, 4, 109-111.

7. Miike I. : Do Insect transmit AIDS. Congress of the United States. Office of technology assessment, 1987, $43 \mathrm{p}$.

8. Mouchet J., Brengues J. : Les interfaces agriculture-santé dans les domaines de l'épidémiologie des maladies à vecteurs et de la lutte antivectorielle. Bull. Soc. Pathol. Exot., 1990, 83, 325-343.

9. Organisation Mondiale de la Santé : Dix années de lutte contre l'onchocercose en Afrique de l'Ouest. OCP/GVA/85.1A, 1985, 137 p. multigr.

10. Rozendaal J. A. : Impregnated mosquito nets and curtains for self-protection and vector control. Trop. Dis. Bull., 1989, $86,41 \mathrm{p}$.

11. Service M. W. (Editor) : Biosystematics of haematophagous insects. Oxford University Press, 1988, $363 \mathrm{p}$.

12. Sigal L. H. : Lyme disease: a world-wide borreliosis. Clin. Exp. Rhumatol., 1988, 6, 411-421. 